\title{
INVESTIGATING THE MECHANISMS OF HOARDING FROM AN EXPERIMENTAL PERSPECTIVE
}

\author{
Stephanie D. Preston, Ph.D., ${ }^{1 *}$ Jordana R. Muroff, Ph.D., ${ }^{2}$ and Steven M. Wengrovitz, M.A. ${ }^{3}$
}

\begin{abstract}
Background: Acquiring and discarding objects are routine decision processes for most people. Despite the ubiquitous need to make such decisions, little is known about bow they are made and what goes wrong when individuals acquire and fail to discard so many items that many areas of their bome become unlivable (i.e., clinical hoarding). We hypothesize that clinical boarding reflects a normal variation in the tendency to acquire and retain objects, only just at a more extreme level. Methods: To test this hypothesis, we examined 89 nonclinical, undergraduate students' performance on a novel experimental paradigm that measures decisions about acquiring and discarding everyday objects. To test our bypothesis, and validate our task as a possible research tool for studying boarding, we related decisions on the task to a variety of measures known to correlate with clinical hoarding. The paradigm was sensitive to individual differences, as subjects varied widely in the quantity of objects they chose to acquire and retain under an increasing pressure to discard. In addition, we replicated expected relationships from the clinical hoarding literature between acquisition and retention tendencies and self-report measures of hoarding, indecisiveness, and obsessive-compulsive behavior. Results: Our data suggest that decisions about objects, even in a nonclinical undergraduate population, vary widely and are influenced by the same variables that influence clinical hoarding, but to a less extreme degree. Conclusions: Future research with this experimental task can separately investigate the role of acquisition, retention, impulsivity, and sensitivity to constraints in clinical boarding to inform our understanding of this disorder. Depression and Anxiety 26:425-437, 2009. (C) 2009 Wiley-Liss, Inc.
\end{abstract}

Key words: decision making; hoarding; OCD; impulsivity; personality; indecisiveness

\begin{abstract}
INTRODUCTION
$\mathbf{M}$ any times a day people must make resourceallocation decisions, such as whether to acquire, maintain, or discard objects to ensure a sufficient supply for future needs without exceeding typical constraints such as physical space, time, and money. This is a complex decision process that seems to be impaired in individuals with clinical "hoarding," where such individuals acquire and do not discard items, even those that are "useless or of limited value," until their homes become so cluttered as to be partially or entirely unlivable and even unsafe. ${ }^{[1]}$ As a clinical disorder,
\end{abstract}

${ }^{1}$ Department of Psychology, University of Michigan, Ann Arbor, Michigan

${ }^{2}$ School of Social Work, Boston University, Boston, Massachusetts

${ }^{3}$ Department of Psychology, Wesleyan University, Middletown, Connecticut

*Correspondence to: Stephanie D. Preston, Ph.D., Department of Psychology, University of Michigan, 530 Church Street, Ann Arbor, MI 48109 USA. E-mail: prestos@umich.edu

Received for publication 26 April 2007; Revised 1 September 2007; Accepted 19 September 2007

DOI 10.1002/da.20417

Published online 25 February 2009 in Wiley InterScience (www. interscience.wiley.com).

(C) 2009 Wiley-Liss, Inc. 
hoarding has been difficult to diagnostically categorize and challenging to treat both because of its complexity and because of our limited understanding of the cognitive processes and biological bases of normal decisions to acquire and discard objects.

Problematic hoarding often results from traumatic insult to the frontal cortex ${ }^{[2,3]}$ and fMRI studies find that hoarders have reduced activation in the dorsal anterior and posterior cingulate cortices, compared to control and nonhoarding obsessive-compulsive disorder (OCD) patients, respectively. ${ }^{[4]}$ In nonclinical populations, normal purchase decisions activate the nucleus accumbens (NAc) in response to pictures of desirable objects. ${ }^{[5]}$ Taken together, the data suggest that decisions about objects, and impairments therein, may be subserved by the dopaminergic mesolimbocortical system, including the ventral tegmentum, NAc, anterior cingulate and frontal cortex.

In rodent species, these same areas are known to be involved in adaptive food-storing behavior in animals, also called "hoarding." The NAc has been associated in animals and humans with the anticipation and motivation toward rewarding stimuli such as drugs and food. ${ }^{[6,7]}$ When the NAc is lesioned in rats, animals continue to consume food normally, and will carry food from the feeding site to a secondary location to consume it, but no longer carry food to a secondary location for storage after they are sated-this impairment is specific to hoarding and is not associated with a reduction in food consumption, activity level, the response to amphetamine, or damage to other forebrain structures; rather, it seems to be because of an inability to be motivated by the rewarding properties of food once the primary cues of motivation (e.g. smell, feel) have been dampened by satiety. ${ }^{[8]}$ The medial frontal cortex, which is linked to the NAc through bidirectional connections in the dopaminergic mesocortical circuit, is also thought to be involved in producing behaviors toward food that are not dependent on the primary features of the food or environment; when the medial frontal cortex is bilaterally ablated in rats, the animals reduce hoarding, treat food items as smaller than they are, and show an increased sensitivity to primary environmental cues such as illumination. ${ }^{[9]}$ Parallel research on the mechanisms of acquiring and retaining objects in nonclinical and clinical populations could greatly advance our understanding of hoarding-a ubiquitous but poorly understood process.

Evidence for a relationship between hoarding tendencies and OCD has been repeatedly found using selfreport measures. Hoarding is commonly diagnosed as a subtype of OCD ${ }^{[10,11]}$ although there are compelling reasons to consider hoarding as distinct from nonhoarding forms of OCD. ${ }^{[2]}$ A hoarder may be diagnosed with OCD in the absence of any additional OCD symptoms, hoarding is associated with different genetic markers than nonhoarding OCD, ${ }^{[13]}$ and it is resistant to traditional pharmacological treatment of
OCD $^{[14-16]}$ (but see Saxena ${ }^{[12]}$ ). Alternatively, behavioral treatment of hoarding in OCD is often shown to be efficacious; ${ }^{[17]}$ Tolin and colleagues conducted an open trial using cognitive behavioral therapy and reported improved hoarding symptoms for half of their 10 hoarding participants. Hoarding also occurs in combination with many other conditions including depression, ${ }^{[10,11,18]}$ anorexia, ${ }^{[19]}$ dementia, ${ }^{[20,21]}$ schizophrenia, ${ }^{[22]}$ social phobia, and personality disorders. ${ }^{[23]}$ Additionally, the presence of compulsive buying ${ }^{[11,24,25]}$ and comorbid impulse control problems like trichotillomania, skin picking, and kleptomania ${ }^{[23]}$ have led some to suggest that hoarding is an impulse-control disorder or exists along a "compulsive-impulsive" spectrum [as defined by McElroy et al. ${ }^{[26]}$. In sum, although hoarding surely is contributed to by anxiety, the situation is complex and further research is needed to determine the etiology of hoarding.

We believe that one reason why hoarding is so poorly understood is that we have very little knowledge on decisions about acquiring and retaining objects in general, whether unimpaired or impaired. By approaching the issue from a cognitive psychology perspective, the present work aims to address this insufficiency in the literature. To understand complex behavior, cognitive psychology breaks processes down into their component parts, assuming that impairments result from a break in one of the subprocesses. Within this framework, hoarding would result from a specific impairment in either assessing the benefits of an object, the likelihood of needing it later, the cost incurred by retaining it, or the integration of these variables to produce a decision. However, a cognitive model may not be productive as an impairment in any one of these subprocesses would ostensibly produce an impairment in the others. For example, hoarders likely are impaired at assessing the benefits of objects, as they perceive objects as more useful or attach sentimental value to a wider range of items than comparison subjects; $;^{[1,27,28]}$ this would also cause items to be perceived as more necessary in the future, which would in turn minimize the cost of retaining them. Unusually high regard for objects could also explain the poor insight of hoarders, because it would be only logical to keep and maintain objects that are so clearly useful or sentimental. ${ }^{[15,29]}$ Although highly explanatory, a simple "overvaluation of objects" model does not directly explain why hoarding is related to anxiety, obsessions and compulsions, or trait indecisiveness. ${ }^{[30]} \mathrm{We}$ argue that it is especially difficult to tease apart the contributing factors to hoarding because we do not understand how people normally make decisions about acquiring and retaining objects.

Even in nonclinical populations, there are substantial deviations in the quantity and quality of objects that people choose to acquire and maintain. For example, some individuals prefer a "homey" aesthetic, full of knick-knacks and memorabilia, whereas others prefer a more Spartan environment, full of "clean lines" and 
absent of clutter. We hypothesize that hoarding is an extreme version of the more "homey" phenotype, drawn from the same distribution but much farther out on the tails, to the point where disorganized piles of clutter can travel as high as the ceiling. As such, the same psychological processes that produce normal variation in object acquisition and retention produce clinical hoarding at the extremes.

As evidence for a link between nonclincial and clinical decisions about acquiring and retaining objects, research in nonclinical populations has found similar relationships between nonclinical hoarding tendencies and clinical hoarding symptoms. ${ }^{[28,29]}$ For example, Frost et al. found that individuals with increased hoarding tendencies also exhibit increased cognitivebehavioral problems including social anxiety, anxiety sensitivity, depressive symptoms ${ }^{[31]}$ obsessive compulsiveness, ${ }^{[31,32]}$ perfectionism, ${ }^{[27]}$ and indecisiveness. ${ }^{[30]}$

One limitation of these studies is that they tend to rely solely on self-report measures of hoarding tendencies, psychopathology and personality. Selfreport measures may be less sensitive than behavioral measures because they are affected by biases such as social desirability and limited by the ability of subjects to understand their own behavior and motivations; ${ }^{[33]}$ this is especially problematic for a disorder like hoarding, which is characterized by poor insight. Moreover, to understand decisions about resources in general, it would be useful to have a behavioral task that can be manipulated and tested in a variety of circumstances, including functional neuroimaging, to test specific hypotheses about the cognitive and neural processes involved. More systematic testing of the reasons why people acquire and retain objects, and why some people do so to an excessive degree, can elucidate clinical hoarding as well as normative decisions about the acquisition and retention of objects.

Toward that end, we used a novel experimental paradigm that separately assessed subjects' understanding of the value of objects, their tendency to acquire various types of free, everyday objects, and their ability to discard acquired items under increasing pressure to conform to space limitations. To determine if increased acquisition and retention in this nonclinical population was driven by similar factors as clinicallevel hoarding, we also correlated behavior on the task with self-report measures of anxiety, depression, obsessions and compulsions, hoarding (acquisition and discarding), indecisiveness, and procrastination. We expected subjects to differ on both the quantity and quality of objects selected, and for how long they would retain the items under pressure to discard. Subjects who took more items were expected to respond faster to acquisition trials and slower to discard trials whereas those who took few items would show the reverse pattern. Subjects who took more items, in keeping with the clinical literature, were also expected to rate items as more useful and exhibit higher (but not necessarily clinical-level) scores on the hoarding scales, obsessions and compulsions, and indecisiveness.

\section{METHODS}

\section{TASK OVERVIEW}

Subjects were presented with a computerized experimental task using pictures of everyday objects that one might find in a home, ranging from objects of no clear value or usefulness (broken egg shells) to very valuable (diamond ring) or very useful (new legal pads) objects. First, the subjects were instructed to acquire any of 107 items that they would (hypothetically) want to take home for free, to measure the tendency to acquire things. This was followed by multiple rounds of discarding the acquired objects, with increasing pressure, to determine how sensitive subjects were to constraints. We used the decisions about objects to mathematically create three clusters of decision makers, based on the quantity and quality of objects that the subjects took. To further validate the experimental task, we used these subject clusters to investigate relationships between the quantity of objects and self-report measures of psychopathology and trait decision making known to be associated with hoarding.

\section{PARTICIPANTS}

This study was performed in the University of Michigan Department of Psychology. The participants were 89 undergraduate students (46 males, 43 females; age range: 17-24) enrolled in introductory psychology at the University of Michigan who received course credit in exchange for their voluntary participation.

\section{GENERAL OVERVIEW}

The study was administered in a PC computer lab with subjects seated at every other computer. After being seated, the subjects read and signed an informed consent document and then completed all elements of the experiment on the computer in a single session lasting approximately $90 \mathrm{~min}$.

There were four main components of the study. The first two, the Object Estimate Task and the Object Decision Task (both described in more detail below), were computerized tasks administered using EPrime Version 1.1 (Psychology Software Tools Inc., Pittsburg, PA); the order of these two tasks was counterbalanced across subjects (half did Estimation first, half Decision first). After completing these two tasks, the subjects completed a series of personality and psychopathology scales (described below and in Table 1). After all the elements were completed, the subjects were debriefed, and remained in their seats until the end of the session.

\section{STIMULI}

Each task used color, digital pictures of real objects taken by a professional photographer against a white background. The pictures were created such that each object occupied approximately the same proportion of the picture and, thus, were not to scale. Bitmap images were presented on a computer screen at $10 \times 10$ centimeters square (original image size: $615 \times 461$, DPI: 96). A pool of 214 pictures was divided into two batches, referred to hereafter as A and B, so that estimations of the objects would not influence decisions about the objects and vice versa. Thus, half of the subjects estimated batch A and made decisions about batch $\mathrm{B}$, whereas the other half estimated batch B and made decisions about batch A. Pilot data were used to create roughly similar distributions of the types of objects in the two batches based on the value, usefulness, and tendency to be saved. 


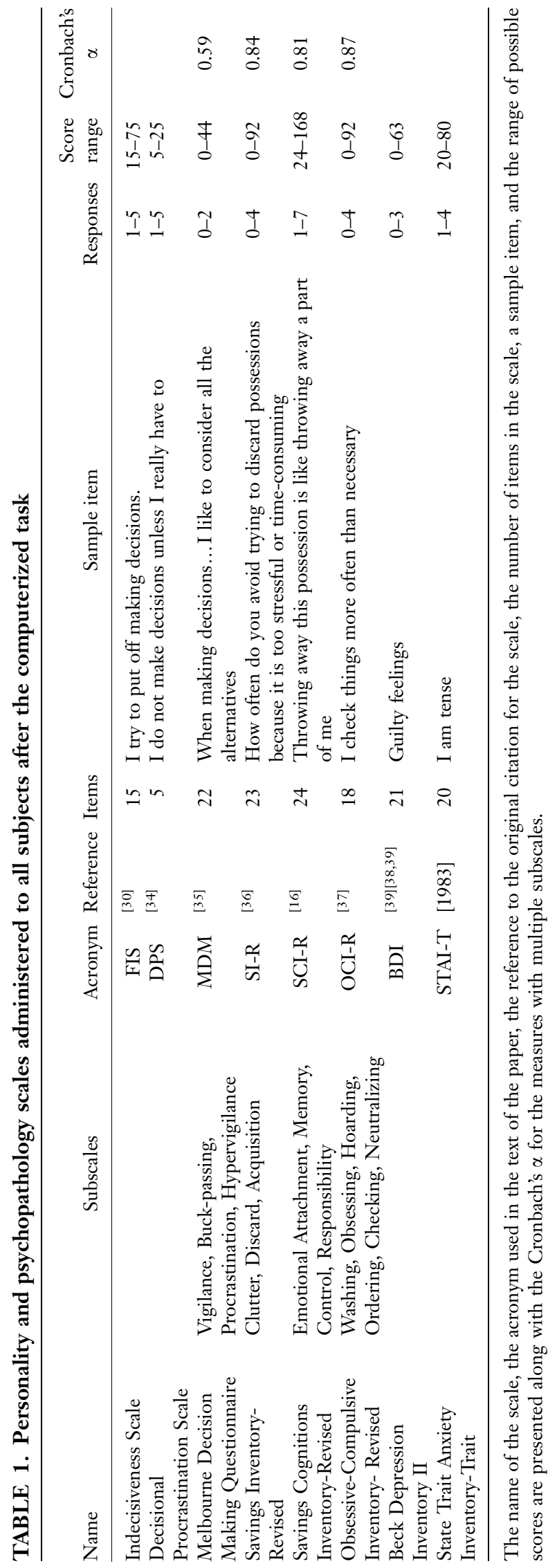


Within each block, the pictures were always presented in randomized order.

\section{OBJECT ESTIMATE TASK}

The Object Estimate Task was comprised of two blocks, order counterbalanced. For the price estimates, subjects viewed each object in their assigned batch (A or B), one at a time, in randomized order, and estimated the price of the object in dollars, using whole numbers $(\$ 0, \$ 1, \$ 2, \$ 3 \ldots)$. For the usefulness estimates, the subjects viewed each object in the same batch, again in random order, and judged how useful the item was on a scale from 1 ("not useful at all") to 7 ("extremely useful").

\section{OBJECT DECISION TASK}

The Object Decision Task is comprised of three parts: exposure to items, personal object decisions, and other's object decisions, in that order. The task was preceded by a practice version that was identical to the test version of the task, but only containing seven pictures. In this way, individuals were exposed to the entire structure of the task and were given a chance to ask questions so that they would understand all phases of the task before starting data collection.

To give each subject sufficient time to decide in the test blocks, but still provide time pressure to decide, each subject was given a variable amount of time to decide per picture in the personal and other object decision parts that was based on how long they took, on average, to make decisions in the practice version. We call this the adaptive-timing algorithm, which we calculated using the following formula:

$$
T_{\text {test }}=M_{\text {practice }}+S D_{\text {practice }}+Z \text {. }
$$

In this formula, $T$ is a variable stimulus presentation time for the test trials that differs as a function of each subject's mean time to respond to each stimulus in the practice version $(M)$, plus their standard deviation for this measure $(S D)$, plus an additional, randomly selected amount of time $(Z)$ added to each trial (between -500 and $500 \mathrm{~ms}$ ) to prevent stereotyped responding.

In the exposure block, subjects viewed all 107 pictures from their assigned batch (A or B; the batch not used in their Object Estimate Task), one at a time, for as long as they wanted to by holding down the spacebar on the keyboard. This allowed the subjects time to view each item freely so that in the subsequent decision blocks the decisions would not be contaminated by confusion about the identity of the object.

The personal object acquisition/retention decision part of the experiment consisted of four blocks of trials: the acquisition block, free discard block, low-pressure discard block, and high-pressure discard block (in that order) in which the subjects were explicitly instructed to "imagine that we have all of these items in the lab, and you can take home whichever ones you want to for free." (No objects were actually transferred.) In the acquisition block, the participants again viewed all 107 pictures in randomized order with a presentation time based on the adaptive-timing algorithm and were asked to press any key if they wanted to keep the item. If they depressed a key before the picture disappeared, a green border appeared around the picture, indicating that they successfully took it. The picture remained up for the duration of the calculated time, at which point, a screen came up asking subjects to press a key to go to the next trial, allowing subjects to rest between pictures if necessary. Following the acquisition phase, the subjects performed a series of discard phases where they were presented with all of the items they chose to acquire in the previous block, in randomized order. First, there was a free discard phase, where they were simply told that they could remove any items that they had previously taken, for example, if they changed their mind or took something by accident. Next, there was a low-pressure block where they "could only keep as many items as would fit in a shopping cart" (hereafter referred to as the "cart" block). Next, there was a high-pressure block where they "could only keep as many items as would fit in a paper bag" (hereafter referred to as the "bag" block; the bag shown was a standard, grocery-store-sized paper bag). It should be noted that these space parameters were not enforced in any way and subjects could keep more items than would actually fit into the allotted space.

Personal discard tendencies are constrained by how many items a subject acquired, creating difficulties in analyzing the discard tendencies because of the great variation in the number of items that could be discarded (i.e., floor effects and heterogeneities in variance). Therefore, an additional block was appended as a pure measure of discard tendencies irrespective of acquisition tendencies. The other's object decision block of the experiment consisted of a single block of 60 pictures from the A and B batch, shown one at a time, in randomized order, in which subjects were explicitly told "these were items that another subject had chosen and you should discard any of their items that you do not want."

\section{QUESTIONNAIRES}

Following the computerized tasks, the participants completed a battery of standard scales measuring psychopathology, hoarding, and trait decision making (Table 1). There were two psychopathology scales. The Beck Depression Inventory $\mathrm{II}^{[39]}$ consists of one total score and was administered because hoarding is known to be affected by depression. ${ }^{[10]}$ The Beck Depression Inventory has good internal consistency and reasonable construct validity. ${ }^{[38]}$ The ObsessiveCompulsive Inventory-Revised is an instrument that was designed to measure obsessions and compulsions on six separate dimensionswashing, obsessing, ordering, checking, neutralizing, and hoarding. ${ }^{[37]}$ The subscales show high internal consistency, moderate to high test-retest reliability, and good convergent validity with other OCD measures. It differentiates well between people with OCD and nonanxious controls. ${ }^{[37]}$ OCD symptoms needed to be assessed because hoarding is currently diagnosed as a form of OCD; this particular instrument was selected because it has been used previously with a nonclinical population and it includes three specific items on hoarding ("I have saved up so many things that they get in the way," "I collect things I don't need," and "I avoid throwing things away because I am afraid I might need them later"). The State-Trait Anxiety Inventory [STAI-Trait version], ${ }^{[40]}$ consisting of one total score, was administered because hoarding is classified as an anxiety disorder. Because this instrument was designed to assess anxiety in nonclinical populations, it may detect anxiety in our sample that a clinical instrument may not. The STAI has good to excellent internal consistency ( $\alpha$ between .86 and .95 ), good test-retest reliability ( $r .76$ to .86), and good convergent validity. There were two hoardingrelated scales that were selected because they have been previously validated for detecting differences in hoarding tendencies in both nonclinical and clinical populations. The Saving Inventory-Revised is a scale designed to measure hoarding tendencies, with separate subscales for acquisition, discard, clutter, and a total score. ${ }^{[36]}$ The three subscales have been shown to have good internal consistency and test-retest reliability. ${ }^{[36]}$ The Saving Cognitions InventoryRevised is a scale designed to look at people's reasons for keeping items including subscales for emotional attachment, memory, control, and responsibility. ${ }^{[41]}$ These subscales showed good internal consistency and convergent and discriminant validity. ${ }^{[41]}$ Hoarding is known to be influenced by indecisiveness; thus, we used three separate scales that measure the ability to make decisions quickly versus slowly. The Frost Indecisiveness Scale has previously been used to study hoarding, consisting of one total score. ${ }^{[30]}$ It has been 
shown to have high internal reliability and is correlated to other hoarding, procrastination, perfectionism, decision making, and compulsivity measures. ${ }^{[30]}$ The Decisional Procrastination Scale is a short instrument that consists of one total score that measures the tendency to put off decisions. ${ }^{[3]}$ It is reliable and valid with a Cronbach $\alpha$ of .80 and 1-month test-retest reliability of .69 [Mann, personal communication, January 5, $\left.1988^{[42]}\right]$. The Melbourne Decision Making Questionnaire is a longer instrument with separate subscales for multiple decision making elements including vigilance, hypervigilance, buck-passing, and procrastination with good psychometric properties. These scales show good reliability with vigilance $(\alpha=0.80)$, hypervigilance $(\alpha=.74)$, buck-passing $(\alpha=0.87)$, and procrastination $(\alpha=0.81) .^{[35]}$

\section{ANALYSIS AND RESULTS}

For ease of presentation and clarity, given the complexity of the analyses, we present the results together with their aforementioned analysis technique, grouped according to the construct of interest. Because half of the subjects viewed batch A pictures and the other half batch B pictures, analyses were first presented separately for the two groups, to determine through descriptive statistics and general analysis of the object decisions if the two groups were really comparable. Then, multidimensional cluster analyses were performed on both groups separately to isolate different types of subjects, resulting in three clusters in each group. Follow-up analyses were performed to determine what differed among the three clusters, and to ensure that the clusters could be combined across the A and B batches. Finally, hypothesis-driven tests were done to determine if the three types of subject clusters differed in their impulsivity, depression, OCD symptoms, anxiety, hoarding, or the ability to make decisions. All the analyses were performed using SPSS (SPSS Inc., Chicago, IL), version 11.0.4 for Macintosh. The $\alpha$ level was set at .05 for all comparisons.

\section{DESCRIPTIVE STATISTICS}

On average, subjects viewed each picture for a mean of $946.57 \mathrm{~ms}(S D=346.94 \mathrm{~ms})$. In the personal object decision part of the task, out of 107 total items, people acquired on average 34.49 items $(S D=17.83$, range: $0-82)$, discarded 5.52 of these in the free-discard block $(S D=4.61$, range: $0-21), 4.42$ more in the cart block $(S D=5.62$; range: $0-30)$, and 8.98 more in the bag block ( $S D=7.94$; range: $0-36)$, for an average sum of 15.58 items retained in the paper bag $(S D=7.73$ range: $0-46)$. In the other's object decision part of the task, out of the 60 total items, people discarded on average 30.21 of the other person's items $(S D=11.14$; range: $0-52)$ for a total of 29.79 items remaining at the end of the block $(S D=11.14$; range: $8-60)$.

Repeated-measures analysis of variance (ANOVA) was used to determine if changes in the number of items kept across the four personal decision blocks (i.e., acquisition, free discard, cart, bag) differed by block, demonstrating that subjects were taking the discard blocks and their space constraints seriously; sex and age were included as independent variables because hoarding is known to be more common in females and with older participants. The number of items kept by subjects did differ significantly by block, $(F(3,186)=63.646, P<.001)$ both for batches A (main effect: $F(1,124)=542.696, P<.001)$ and $\mathrm{B}$ (main effect: $F(1,130)=253.617, P<.001)$, due to an overall decrease in the number of items kept as the blocks progressed in both the batches (A: $-13.59, t=-23.30$, $P<.001$; B: $-9.60, t=-16.44, P<.001)$; this demonstrates that, overall, subjects responded adaptively to the increasing space constraints. The number of objects in each block did not differ by age $(F(7,89)=0.923$, ns) or sex $(F(1,76)=1.549, \mathrm{~ns})$, and there was no interaction $(F(4,76)=1.528, \mathrm{~ns})$.

\section{SUBJECT CLUSTERS}

Statistical determination of the three clusters. Behavior on the Object Decision Task varied widely across subjects, but pilot data suggest at least three common strategies: taking many items and discarding very few, taking very few items and not discarding, and taking many items but also discarding a lot under pressure. To search for these expected commonalities among subjects, hierarchical cluster analyses were performed using the Ward method ${ }^{[43]}$ on the number of blocks each item was kept (0-4) in the personal object decision part of the experiment. The Ward method for cluster analysis starts with each individual as its own cluster, and then computes the geometric distance between subjects in multidimensional space. Clusters are progressively merged to create larger and larger clusters by minimizing the Sum of Squares based on the distance between subjects, until three groups are formed (a stable and significant number of groups that we prespecified based on pilot data).

As an input to the cluster analysis, we created a new variable that encapsulated three different aspects of the subjects' performance into a single variable-the "duration" data. The duration variable represented the number of blocks that each subject kept each object, from 0 (never taken) to 4 (kept through the high-pressure block). Importantly then, the statistical grouping of subjects was based on a combination of the number and identity of items acquired and how long each was retained across the blocks. This multidimensional variable is more powerful than looking at a single variable (such as the number acquired), which may not effectively represent the underlying construct of interest.

Because the duration data included object identity information and the $\mathrm{A}$ and $\mathrm{B}$ batches consisted of different objects, cluster analyses were initially done separately for the A and B groups. We selectively looked for three-cluster solutions in each group and found that solution to be statistically significant in both the groups. 


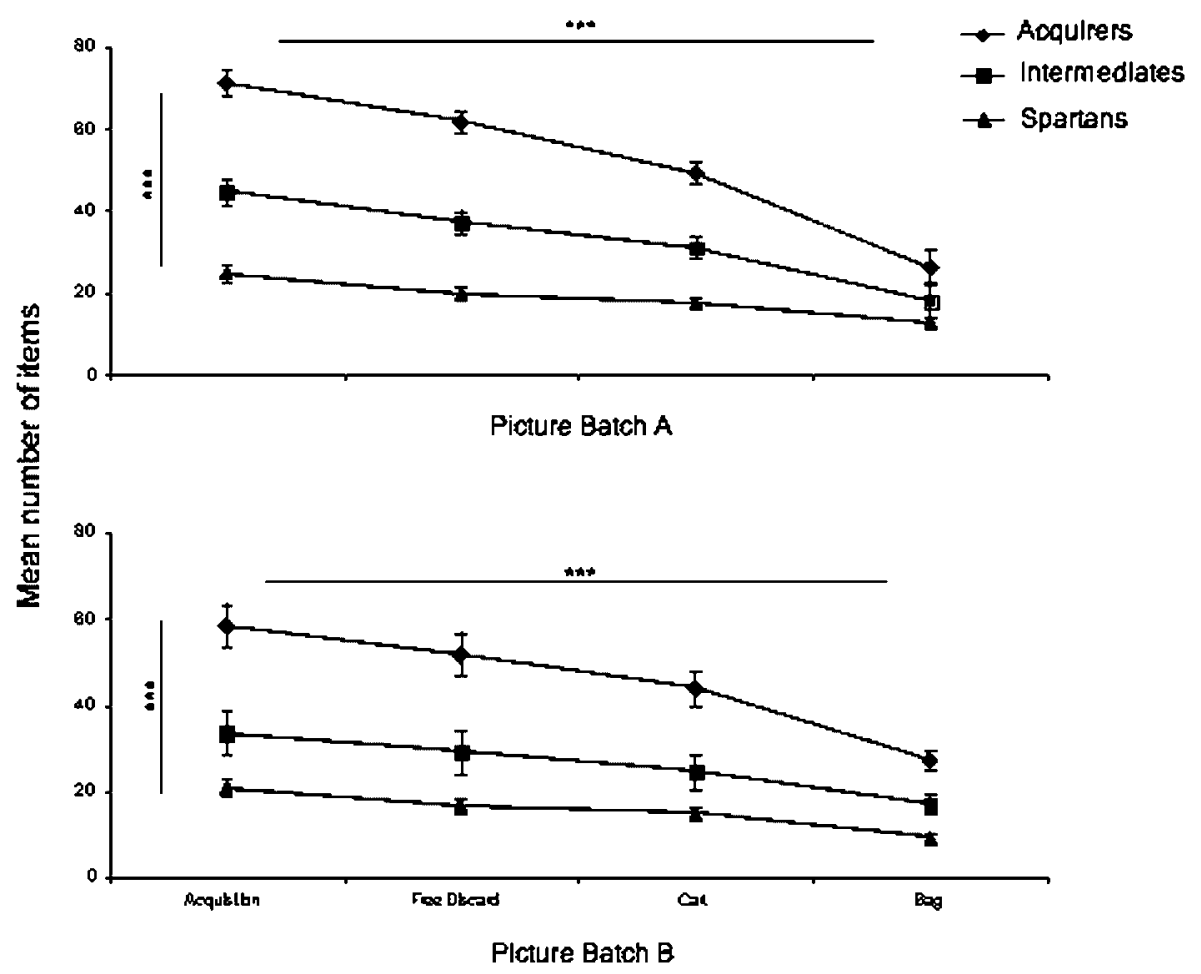

Figure 1. (a and b) average number of items selected ( \pm standard error) for batches A and B, respectively, by block and subject cluster. Diamonds represent Acquiring cluster, squares represent Intermediate cluster, and triangles represent Spartan cluster. Asterisks denote significant differences $\left({ }^{* * *} P<.001\right)$.

Follow-up analyses to interpret the three clusters. Cluster analysis is agnostic with respect to the interpretation of the clusters and "duration" is a multidimensional variable. Therefore, follow-up analyses were required to determine what exactly differed among the subject clusters. Specifically, we planned to look at the amount acquired and the rate of discard to determine what caused the subjects to be grouped this way and if the grouping was the same for the two batches of subjects (A and B).

For each of the follow-up dependent measures (number of personal items acquired and discarded and number of other's objects discarded), we modeled differences among the three subject clusters using a general linear model, run separately for batches A and B. Subject identity was modeled as a random factor (using the iterative restricted maximum likelihood methods), subject cluster as a nominal factor, block number as a continuous factor (four levels), and the cluster-by-block interaction was included to look for significant effects in the contrasts of block, cluster, and a block-by-cluster interaction. Scaled estimates were used to further investigate significant interactions.

If the results from these general linear models are the same in the A and B batches, then the subjects who viewed batch $A$ and those who viewed batch $B$ pictures can be combined into one larger set of three clusters and these larger groups can be investigated to test specific hypotheses. For example, we could determine from the data collected in the task if the subjects who kept more objects were more impulsive (took things faster), estimated objects as being worth more money or more useful, or had higher rates of self-reported depression, anxiety, hoarding, indecisiveness, and procrastination.

Acquisition decisions. A main effect of the number of items taken per cluster was observed in batches A $(F(2,12)=72.434, \quad P<.001)$ and $\mathrm{B}$ $(F(2,127)=58.275, P<.001)$ (Fig. 1a and b). In each batch, significantly more objects than average were taken by one group, hereafter the "Acquiring cluster" (A: 17.62, $t=9.38, \quad P<.001 ; \quad \mathrm{B}: 16.42, t=9.64$, $P<.001)$, significantly fewer objects than average were taken by another group, hereafter the "Spartan cluster" (A: $-15.82, t=-11.66, P<.001$; B: $-13.27, t=-9.7$, $P<.001$ ), and a third group was intermediate, hereafter the "Intermediate cluster." Fewer objects than average were taken by the Intermediate cluster in batch B only (A: $-1.80, t=-1.18$, ns; B: $-3.15, t=-2.25, P<.05$ ).

Discard decisions. The block-by-cluster interaction was significant in batch A $(F(2,124)=66.261$, $P<.001)$ and batch $\mathrm{B}(F(2,127)=18.988, P<.001)$, due to the fact that significantly more items than average were discarded per block by the Acquiring cluster (A: $-8.53, t=-8.82, \quad P<.001 ; \quad \mathrm{B}:-5.65, t=-5.86$, $P<.001)$, significantly fewer objects than average were discarded per block by the Spartan cluster (A: 7.84, $t=11.21, P<.001 ;$ B: $3.92, t=5.06, P<.001)$, and 
significantly fewer objects than average were discarded per block by the Intermediate cluster in batch B (A: 0.69 , ns.; B: $1.52, t=1.73 P<.05)$. Note, these effects are due to the fact that the Acquiring cluster started with many more items, requiring that many more be discarded to comply with the decreasing space; these effects remain even when analyses are done using the percentage of items held, rather than the raw number $(F>3.62,|t|>2.28, P<0.05)$.

Other's object decisions. Univariate ANOVA was used to compare the number of items discarded in the other's object decision block for each of the subject clusters, again separately for batches A and B.

There were no significant differences between subject clusters for the number of items discarded during the other's object decision part of the task for batch A $(F(2,40)=1.647$, ns $)$ or batch B $(F(2,43)=2.452$, ns).

\section{THE ACQUIRING, INTERMEDIATE, AND SPARTAN CLUSTERS}

In the previous analyses, we determined that there were some systematic differences between the three subject clusters that were mirrored in the two pictures batches, A and B. That is, in both the A and the B groups, there were three clusters of subjects with the following properties: (1) one cluster of subjects took many more things and subsequently had to discard many more things to try to meet the increasing space constraints (hereafter the Acquiring cluster), (2) one group took very few things and therefore did not have to remove items to meet the increasing space constraints (hereafter the Spartan cluster), and (3) one group shared properties of both the Acquiring and Spartan cluster, taking more items initially, but then discarding more items to meet the constraints, so that they took many fewer items total and were more successful (than the Acquiring cluster) in meeting the space constraints (hereafter, the Intermediate cluster). For increased statistical power with our subsequent analyses, we combined the respective clusters from A and $\mathrm{B}$ into three larger clusters and used them to investigate hypothesized contributors to acquiring and retaining items (gender, impulse control problems, overvaluation of price or usefulness, hoarding tendencies, depression, anxiety, obsessions and compulsions, and decision impairment).

Gender frequencies by subject cluster. Because of the fact that clinical hoarding studies find a greater proportion of female hoarders than male, $\chi^{2}$ was used to determine if there were more females in our Acquiring cluster. There were no significant differences in the number of males and females across the three clusters in the three combined subject clusters $\left(\chi^{2}(2, N=89)=4.147, P=.126\right)$; although nonsignificant, there were about twice as many females as compared to males in the Acquiring cluster in batch $\mathrm{A}$, batch $\mathrm{B}$, and the combined group (Table 2).
TABLE 2. Frequencies of individuals in each of the three clusters (the Spartan cluster, Intermediate cluster, and Acquiring cluster) broken down by gender within each picture batch ( $A$ and $B$ ) and for the three combined subject clusters

\begin{tabular}{lcccc}
\hline & & \multicolumn{3}{c}{ Cluster } \\
\cline { 3 - 5 } Picture batch & Gender & Acquiring & Intermediate & Spartan \\
\hline A & Female & 5 & 6 & 11 \\
& Male & 1 & 7 & 13 \\
B & Total & 6 & 13 & 24 \\
& Female & 5 & 6 & 10 \\
A and B combined & Male & 3 & 12 & 10 \\
& Total & 8 & 18 & 20 \\
& Female & 10 & 12 & 21 \\
& Male & 4 & 19 & 23 \\
& Total & 14 & 31 & 44 \\
\hline
\end{tabular}

Impulse control by subject cluster. To investigate possible differences in impulse control across the three clusters, we compared mean response times across the four personal response blocks using repeated-measures ANOVA for the combined subject clusters. Note that only objects acquired in the acquisition block and discarded in the discard blocks yielded response time data as nonselected items simply disappeared after the duration determined by the adaptive-timing algorithm.

Response times to the acquired and discarded objects differed significantly by block (main effect: $F(3$, $54)=25.373, \quad P<.001$; Fig. 2) owing to shorter response times in the acquire and bag blocks than in the free discard and cart blocks $(t(59)> \pm 3.47$, $P<.001)$. These response times by block also differed as a function of the three combined subject clusters (interaction: $F(6,110)=2.240, P=.045$ ), because the Acquiring cluster responded especially quickly in the acquire block $(M=795.620 \mathrm{~ms})$ whereas the Spartan cluster responded especially slowly in the cart block $(M=1252.256 \mathrm{~ms})$ compared to the global mean $(M=1040.633 \mathrm{~ms})$; however, these pairwise differences were not significant after correcting for multiple comparisons using Tukey's Honestly Significant Differences (HSD) post hoc test $(P>.162)$.

Estimates of value by subject cluster. To investigate possible differences in the perceived value of objects across the three combined subject clusters, multivariate ANOVA was used to determine if the mean $Z$-scores for estimates of price and usefulness in the Object Estimate Task differed across the combined subject clusters.

There were no significant differences among the three combined subject clusters in their relative estimations of price $(F(2,86)=.638, P=.531)$ or usefulness $(F(2,86)=2.100, P=.129)$, although usefulness indicated a trend with the Acquiring cluster having higher positive $Z$-scores for usefulness compared to the 


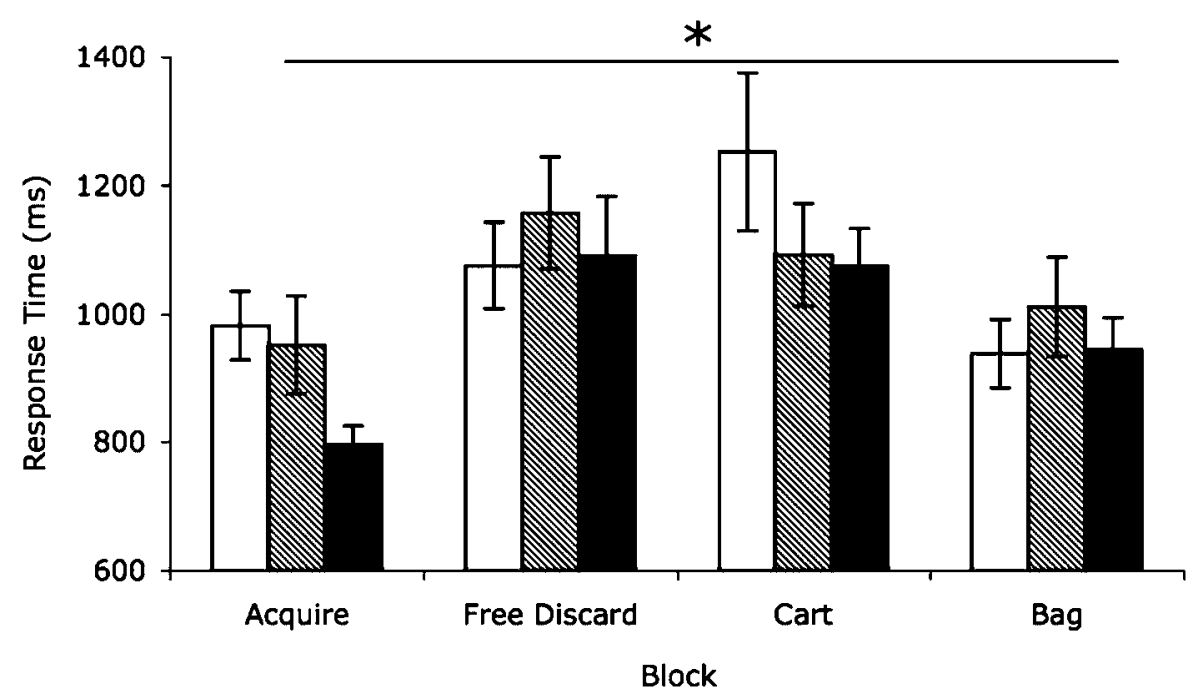

Figure 2. Average response time in milliseconds ( \pm standard error) by block and subject cluster. Unfilled bars represent Spartan cluster, hatched bars represent Intermediate cluster, and filled bars represent Acquiring cluster. The asterisk denotes a significant main effect of block and a block-by-cluster interaction $\left({ }^{*} P<.05\right)$.

Intermediate and Spartan cluster (respectively: $M=0.210,0.015,-0.077)$.

Differences in self-report measures by subject cluster. Multivariate ANOVA was used to determine if there were significant omnibus relationships among the combined subject clusters and their scores on each of the self-report questionnaires. Tukey's HSD post hoc tests were used to determine which of the clusters were significantly different from the others on each scale.

Results from multivariate ANOVA (Table 3) indicate significant differences among the three combined subject clusters for Savings Inventory-Revised Total, Savings Inventory-Revised Discard, Savings InventoryRevised Acquisition, Indecisiveness Scale, Melbourne Decision Making Questionnaire Total, Obsessive Compulsive Inventory-Revised (OCI-R) Total, and OCI-R Checking. Interestingly, on all of these scales the Acquiring cluster scored the highest, meaning that they had more trouble discarding items and more obsessions and compulsions overall, especially related to checking (see Table 3 for detailed pairwise information). The OCI-R Hoarding subscale showed a trend for a difference among the three combined subject clusters $(F(2,82)=2.828, P=.065)$ due to higher mean scores for the Acquiring cluster. No other differences were significant.

\section{DISCUSSION}

A novel experimental paradigm with behavioral dependent measures was used to investigate differences in acquisition and discard behavior among individuals in a nonclinical, undergraduate population. The paradigm proved to be sensitive to individual differences, as it produced significant variations in behavior both for acquisition and discard tendencies, despite the fact that all decisions were explicitly hypothetical (no objects were actually exchanged), and an exclusively nonclinical population was interviewed.

Our population was decidedly homogeneous, with all subjects being approximately at the same age and level of education (and likely other sociodemographics); therefore, although our results may not fully generalize to the general population, the fact that our task found variability in this restricted population is a testament to its sensitivity. Moreover, the fact that we found several standard relationships between the behavior on our task and self-report measures of hoarding, psychopathology, and trait decision making suggests both that nonclinical increases in acquisition and retention may mirror the more extreme increases found in clinical hoarding and that our task is likely to be sensitive to real-world problems making decisions about objects.

Based on the behavior of subjects on the task, we used multidimensional cluster analysis to statistically group subjects into three types, the Acquiring cluster (those who selected and retained the most items), the Spartan cluster (those who selected and retained the fewest items), and the Intermediate cluster (those who selected an intermediate number of items, but also discarded many); these three clusters were then used as an independent variable with three levels to investigate the relationship between acquisition and retention decisions, psychopathology, and trait decision making. The Acquiring cluster took between 42 and 82 of the 107 objects in the initial acquisition block, whereas the Spartan cluster took between 0 and 41, creating completely nonoverlapping distributions in the quantity of objects taken by these groups. These differences 
TABLE 3. Results from the multivariate ANOVA looking at the relationship between the three subject clusters (subjects combined from batches $\mathbf{A}$ and $\mathbf{B}$ ) and their respective scores on the self-report scales $\left({ }^{*} P<.05,{ }^{\mathrm{t}} P<.10\right)$

\begin{tabular}{|c|c|c|c|c|c|}
\hline \multirow[b]{2}{*}{ Scale measure } & \multirow[b]{2}{*}{$F(2,82)$} & \multirow[b]{2}{*}{$P$} & \multicolumn{3}{|c|}{ Cluster } \\
\hline & & & Acquiring & Intermediate & Spartan \\
\hline SI-R Total & 3.241 & $0.044^{*}$ & $31.79(3.53)^{\mathrm{a}}$ & $21.61(2.37)^{\mathrm{b}}$ & $22.29(2.04)^{\mathrm{a}, \mathrm{b}}$ \\
\hline SI-R Clutter & 1.125 & 0.330 & $8.86(1.51)$ & $6.32(1.01)^{\mathrm{a}}$ & $6.45(0.87)$ \\
\hline SI-R Discard & 3.597 & $0.032^{*}$ & $12.07(1.47)^{\mathrm{a}}$ & $7.81(0.98)^{\mathrm{b}}$ & $7.76(0.85)^{\mathrm{b}}$ \\
\hline SI-R Acquisition & 3.501 & $0.035^{*}$ & $10.86(1.08)^{\mathrm{a}}$ & $7.48(0.73)^{\mathrm{b}}$ & $8.07(0.62)^{\mathrm{a}, \mathrm{b}}$ \\
\hline BDI Total & 0.747 & 0.477 & $11.50(2.33)$ & $8.71(1.56)$ & $10.90(1.34)$ \\
\hline BDI Depression & 0.352 & 0.705 & $0.43(0.21)$ & $0.32(0.14)$ & $0.48(0.12)$ \\
\hline FIS & 3.404 & $0.038^{*}$ & $45.79(2.39)^{\mathrm{a}}$ & $38.32(1.60)^{\mathrm{b}}$ & $41.17(1.38)^{\mathrm{a}, \mathrm{b}}$ \\
\hline MDM total & 4.294 & $0.017^{*}$ & $25.29(1.60)^{\mathrm{a}}$ & $19.87(1.07)^{\mathrm{b}}$ & $20.50(0.92)^{\mathrm{b}}$ \\
\hline MDM Vigilance & 1.229 & 0.298 & $10.00(0.61)$ & $9.19(0.41)$ & $8.90(0.35)$ \\
\hline MDM Buck-passing & 2.581 & $0.082^{\mathrm{t}}$ & $6.14(0.69)$ & $4.26(0.46)$ & $4.95(0.40)$ \\
\hline MDM Procrastination & 2.346 & 0.102 & $4.36(0.56)$ & $2.90(0.37)$ & $3.33(0.32)$ \\
\hline MDM Hypervigilance & 2.553 & $0.084^{\mathrm{t}}$ & $4.79(0.57)$ & $3.52(0.38)$ & $3.31(0.33)$ \\
\hline DPS & 2.530 & $0.086^{\mathrm{t}}$ & $14.93(1.06)$ & $12.26(0.71)$ & $12.38(0.61)$ \\
\hline STAI-T & 0.772 & 0.466 & $43.21(2.63)$ & $39.74(1.76)$ & $39.60(1.52)$ \\
\hline SCI-R-Total & 0.973 & 0.382 & $74.14(6.51)$ & $64.06(4.38)$ & $70.05(3.76)$ \\
\hline SCI-R Emotional Attachment & 0.652 & 0.524 & $27.93(3.05)$ & $25.03(2.05)$ & $27.95(1.76)$ \\
\hline SCI-R Memory & 1.388 & 0.255 & $15.21(1.64)$ & $12.03(1.10)$ & $13.57(0.95)$ \\
\hline SCI-R Control & 0.163 & 0.850 & $11.14(1.11)$ & $11.90(0.74)$ & $11.67(0.64)$ \\
\hline SCI-R Responsibility & 2.311 & 0.106 & $19.86(1.85)$ & $15.10(1.24)$ & $16.86(1.07)$ \\
\hline OCI-R-Total & 3.454 & $0.036^{*}$ & $20.29(2.94)^{\mathrm{a}}$ & $16.13(1.97)^{\mathrm{a}, \mathrm{b}}$ & $11.88(1.70)^{\mathrm{b}}$ \\
\hline OCI-R Washing & 0.844 & 0.434 & $2.14(0.56)$ & $1.39(0.37)$ & $1.33(0.32)$ \\
\hline OCI-R Obsessing & 2.113 & 0.127 & $3.29(0.62)$ & $2.90(0.42)$ & $2.02(0.36)$ \\
\hline OCI-R Hoarding & 2.828 & $0.065^{\mathrm{t}}$ & $5.07(0.72)$ & $3.39(0.48)$ & $3.12(0.42)$ \\
\hline OCI-R Ordering & 3.060 & $0.052^{\mathrm{t}}$ & $3.57(0.75)$ & $4.00(0.50)$ & $2.40(0.43)$ \\
\hline OCI-R Checking & 3.348 & $0.04^{*}$ & $3.43(0.57)^{\mathrm{a}}$ & $2.48(0.38)^{\mathrm{a}, \mathrm{b}}$ & $1.79(0.33)^{\mathrm{b}}$ \\
\hline OCI-R Neutralizing & 3.022 & $0.054^{\mathrm{t}}$ & $2.79(0.58)$ & $1.97(0.39)$ & $1.21(0.34)$ \\
\hline
\end{tabular}

Values under each cluster represent means $(S E)$ and letters indicate differences among clusters in Tukey post hoc tests of significance $\left({ }^{*} P<.05\right)$. ANOVA, analysis of variance; SI-R, Savings Inventory-Revised; BDI, Beck Depression Inventory; FIS, Indecisiveness Scale; MDM, Melbourne Decision Making Questionnaire; DPS, Decisional Procrastination Scale; STAI-T, State Trait Anxiety Inventory-Trait; SCI-R, Savings Cognitions Inventory-Revised; OCI-R, Obsessive Compulsive Inventory-Revised.

in acquisition are interesting and are not necessitated by the fact that we isolated three subject groups. The three subject groups were statistically derived based on a multidimensional variable ("duration") that included information about decisions across all four blocks as well as the identity of the objects. As such, subject clusters could have been based on any combination of information about acquisition, rate of discard, or the types of items that were selected or discarded. There was no a priori reason to expect subjects to differ in the acquisition block alone; subjects in the three groups could have simply selected different objects or kept them for longer after having acquired the same amount. Thus, even in our nonclinical sample, the task was able to produce an effective scale and distribution of initial responses to the objects.

The trend for higher usefulness estimates by the Acquiring cluster suggests that they may perceive more objects as useful than their peers; further research with a larger sample can verify this effect and determine if it is due to a greater sense of creativity, flexibility, or vision on the part of the Acquiring cluster, ${ }^{[44]}$ or simply a lower threshold for what they would consider a realistic use. In contrast, it is unlikely that the Acquiring cluster is impaired at detecting the monetary value of objects; three separate experiments (Preston, unpublished) have not found a relationship between the estimated price of objects and object decisions. This is of theoretical importance because it indicates that decisions about objects in the lab, by and large, are based more on the subjective, perceived value and usefulness of objects to the individual than to monetary concerns, the latter of which is emphasized by traditional economic models of decision making. It is possible that something about the hypothetical nature of our task does not allow us to access an existing monetary overvaluation of objects; however, we believe that this hypothesis is unlikely. All other comparisons produced intersubject variability and expected relationships with other measures, demonstrating that the hypothetical nature of the task was not a problem; we do not see any theoretical reason why estimations of price would differ in this regard.

The Acquiring cluster not only wanted the greatest quantity of items, but they also did so with the fastest 
response times in the whole experiment-significantly less than a second, which was faster than the Spartan or Intermediate clusters at any stage. These data support a hypothesized link between hoarding and either compulsiveness or impulsivity. ${ }^{[11,25]}$ However, because the Acquiring cluster responded quickly to acquisition decisions and the Spartan cluster to discard decisions, these effects cannot be due to any generalized personality or motor differences among the Acquiring and Spartan clusters, such as higher enthusiasm, faster decision making, or a compulsive tendency to push buttons. Rather, these differences reveal expected differences in the way that decisions are processed depending on the frame (to acquire an object, or get rid of it). It is also possible that the Acquiring cluster responded quickly to acquisition decisions not because they are impulsive, but simply because they perceived the items as more clearly desirable or useful, and thus were motivated to acquire the objects and were able to respond quickly to a more straightforward choice. Further research is needed to determine the source of these differences in response time.

The Acquiring cluster did discard significantly more objects than the other groups (demonstrating that they were somewhat sensitive to constraints), but overall their compliance was minimal, with 16-46 items in the paper bag, overlapping little with the other two groups who kept between 1 and 26 items. Thus, statistically significant differences in the discard behavior of the Acquiring cluster are mostly attributable to the fact that they simply had more items to dispose of and not to their exceptional ability to discard. One could argue that the incomplete response of the Acquiring cluster to comply with the constraints was owing to the fact that they were not enforced. Debriefing of the subjects, however, revealed a belief in the Acquiring cluster that the constraints did not pose a logistical problem because they could always stack objects high above the rim, use a backpack they acquired to hold some of the objects, or place some objects in the building temporarily until they could get them home [akin to temporary caching near an ephemeral patch of food in nonhuman animals]. ${ }^{[45]}$ While again these responses reflect a logical and creative solution to a problem if taken at the level of the individual decision, hoarding is considered a clinical disorder because such individual decisions, which are always biased toward retention, "stack up," producing long-term situations that have severe negative consequences.

In contrast to the inference that the Acquiring cluster has a general impairment in discarding objects, there were no differences among the three clusters when discarding objects that belonged to someone else (i.e., the other's object decision block). This may suggest that the Acquiring cluster really does have a specific problem with acquisition, ${ }^{[2]}$ with controlling impulses, ${ }^{[1,25]}$ and/or a specific fear of losing an item classified as their own, the latter of which is consistent with the hoarders' increased sentimentality for and emotional comfort from objects. ${ }^{[28]}$ An impairment that is specific to the Acquiring cluster's own objects is also consistent with recent research demonstrating a specific categorization impairment for hoarders when dealing with personal items. ${ }^{[46]}$ Note that the tendency to deem objects as personal would have to occur at a very superficial level as the objects "acquired" in this task were only hypothetical and were never held by the subjects as they are in traditional tests of the Endowment Effect where the subjects increase their valuation of an object, such as a mug, after it is given to them. ${ }^{[47,48]}$

Demonstrating the validity of the experimental task, performance on the task correlated with scale measures of psychopathology and trait decision making, which have been previously shown to be related to hoarding in nonclinical populations ${ }^{[31]}$. The Acquiring cluster had higher scores overall, and on the Acquire and Discard subscales, on the Saving Inventory-Revised-a scale which has been previously shown to relate to realworld hoarding impairment. ${ }^{[27,36]}$ The Acquiring cluster also had higher scores on the Indecisiveness Scale, another measure previously shown to relate to hoarding ${ }^{[27,30,41]}$, and on the more general Melbourne Decision Making Questionnaire, further confirming that hoarders have a decision impairment that extends beyond decisions about objects.

The Acquiring cluster also demonstrated more obsessions and compulsions, reflected in higher total scores on the OCI-R, more checking behavior, as well as trends for more hoarding and neutralizing. Although the OCI-R Hoarding subscale was expected to be the most relevant, checking has also been related to hoarding in earlier research. ${ }^{[30]}$ This further demonstrates the validity of our task and verifies that nonclinical decisions about acquiring and retaining objects are affected by similar underlying constructs. The fact that we observed relationships on these scales designed to test clinical-level problems attests to the strength of the effects and the continuous nature of the phenomenon. Relationships between performance on the task and self-report measures of obsessions and compulsions also lend credence to the view of clinical hoarding as a spectrum disorder of $\mathrm{OCD},{ }^{[26]}$ and a disorder that is emotional as well as cognitive.

Using a traditional decision-making model where costs and benefits, integrated to produce a decision, may not be particularly generative for the study of hoarding because clinical and nonclinical individuals with hoarding tendencies seem to be impaired on most aspects of decision making. It is possible that these problems stem from a more basic problem of viewing objects as much more desirable than persons without hoarding tendencies. This would make it harder to resist the temptation not to take an item, leading to having many more objects and making it incredibly difficult to discard objects both because they are so appealing and because there are a daunting number of 
decisions to be made. Despite its parsimony, this view does not directly capture the salient and empirically validated distress in hoarders related to making a mistake. ${ }^{[27]}$

Perhaps one could take a functional-neuroanatomic approach whereby hoarders would have an underlying problem with their dopaminergic mesolimbocortical system that produces exacerbated negative feedback to unexpected punishment (such as having been caught without an item when one should have had it). This emotional memory, in turn, generates an unusually positive anticipatory signal when contemplating acquiring an object of future use, and an inversely proportional, negative anticipatory signal when contemplating discarding something that may be useful later. This view is consistent with the fact that dysfunction of the orbitofrontal and anterior cingulate cortices (which make up the cortical and limbic portion of this circuit, respectively) is associated with impulse control problems, poor insight, perfectionism, indecisiveness, and anxiety; moreover, the frontal and the striatal regions of this system are associated in lesion or functional imaging studies with hoarding in humans ${ }^{[2-4]}$ and animals ${ }^{8,99}$. Further research on hoarding is needed to specifically link different aspects of the decision process to their respective regions within this system. With this valid experimental task, we can systematically investigate the neural substrates associated with each of the subprocesses of the decision using tools such as functional imaging, event-related potentials, psychophysiology, and transcranial magnetic stimulation.

Hoarding can cause great distress to the individuals involved and their families and creates a public health threat to the community; at the same time, it is difficult to treat or reverse. ${ }^{[49,50]}$ Moreover, even nonclinical consumerism negatively impacts the environment by increasing pollution, waste, and financial and health disparities between high- and low-income groups. ${ }^{[51]}$ More empirical research into the mechanisms of decisions about resources is needed to address these problems. This novel task for experimentally investigating decisions about the acquisition/retention of objects can be particularly effective toward this end because it can access and be used to manipulate multiple aspects of the decision process independently, and thus interrogate the complex interaction between decisions and emotions in a tractable and valid way.

Acknowledgments. The authors would like to acknowledge Amy Paine for help with data collection, Adam Bernstein for programing and data processing, R. Brent Stansfield for analysis, and J. Frank Yates, Andrea Angott, Lydia Chen, and James Abelson for comments on a previous version of this study. Three reviewers also made helpful suggestions for revising a previous version of this study.

\section{REFERENCES}

1. Frost RO, Hartl TL. A cognitive-behavioral model of compulsive hoarding. Behav Res Ther 1996;34:341-350.

2. Anderson SW, Damasio H, Damasio AR. A neural basis for collecting behaviour in humans. Brain 2005;128:201-212.

3. Hahm DS, Kang Y, Cheong SS, Na DL. A compulsive collecting behavior following an A-com aneurysmal rupture. Neurology 2001;56:398-400.

4. Saxena S, Brody AL, Maidment KM, Smith EC, Zohrabi N, Katz E, Baker SK, Baxter Jr. LR Cerebral glucose metabolism in obsessive-compulsive hoarding. Am J Psychiatry 2004;161: $1038-1048$.

5. Knutson B, Rick S, Wimmer GE, Prelec D, Loewenstein G. Neural predictors of purchases. Neuron 2007;53:147-156.

6. Berridge KC, Robinson TE. Parsing reward. Trends Neurosci 2003;26:507-513.

7. Robbins TW, Everitt BJ. Neurobehavioural mechanisms of reward and motivation. Curr Opin Neurobiol 1996;6:228-236.

8. Whishaw IQ, Kornelsen RA. Two types of motivation revealed by ibotenic acid nucleus accumbens lesions: dissociation of food carrying and hoarding and the role of primary and incentive motivation. Behav Brain Res 1993;55:283-295.

9. Whishaw IQ, Oddie SD. Qualitative and quantitative analyses of hoarding in medial frontal cortex rats using a new behavioral paradigm. Behav Brain Res 1989;33:255-266.

10. Frost RO, Steketee G, Williams LF, Warren R. Mood, personality disorder symptoms and disability in obsessive compulsive hoarders: a comparison with clinical and nonclinical controls. Behav Res Ther 2000;38:1071-1081.

11. Winsberg ME, Cassic KS, Koran LM. Hoarding in obsessivecompulsive disorder: a report of 20 cases. J Clin Psychiatry 1999;60:591-597.

12. Saxena S. Is compulsive hoarding a genetically and neurobiologically discrete syndrome? Implications for diagnostic classification. Am J Psychiatry 2007;164:380-384.

13. Samuels J, Shugart YY, Grados MA, Willour VL, Bienvenu OJ, Greenberg BD, et al. Significant linkage to compulsive hoarding on chromosome 14 in families with obsessive-compulsive disorder: Results from the OCD Collaborative Genetics Study. Am J Psychiatry 2007;164:493-499.

14. Mataix-Cols D, Rauch SL, Manzo PA, Jenike MA, Baer L. Use of factor-analyzed symptom dimensions to predict outcome with serotonin reuptake inhibitors and placebo in the treatment of obsessive-compulsive disorder. Am J Psychiatry 1999;156: 1409-1416.

15. Saxena S, Maidment KM. Treatment of compulsive hoarding. J Clin Psychol 2004;60:1143-1154.

16. Steketee G, Frost R. Compulsive hoarding: current status of the research. Clin Psychol Rev 2003;23:905-927.

17. Tolin DF, Frost RO, Steketee G. An open trial of cognitivebehavioral therapy for compulsive hoarding. Behav Res Ther 2007;45:1461-1470.

18. Mancini F, D’Olimpio F, Del Genio M, Didonna F, Prunetti E. Obsessions and compulsions and intolerance for uncertainty in a non-clinical sample. J Anxiety Disord 2002;16:401-411.

19. Frankenburg FR. Hoarding in anorexia nervosa. Br J Med Psychol 1984;57:57-60.

20. Finkel SI, Costa e Silva J, Cohen G, Miller S, Sartorius N. Behavioral and psychological signs and symptoms of dementia: a consensus statement on current knowledge and implications for research and treatment. Int Psychogeriatr 1996;8:497-500.

21. Hwang JP, Tsai SJ, Yang CH, Liu KM, Lirng JF. Hoarding behavior in dementia: a preliminary report. Am J Geriatr Psychiatry 1998;6:285-289. 
22. Luchins DJ, Goldman MB, Lieb M, Hanrahan P. Repetitive behaviors in chronically institutionalized schizophrenic patients. Schizophr Res 1992;8:119-123.

23. Samuels J, Bienvenu III OJ, Riddle MA, Cullen BA, Grados MA, Liang KY, et al. Hoarding in obsessive compulsive disorder: results from a case-control study. Behav Res Ther 2002;40:517-528.

24. Frost RO, Kim HJ, Morris C, Bloss C, Murray-Close M, Steketee G. Hoarding, compulsive buying and reasons for saving. Behav Res Ther 1998;36:657-664.

25. Greenberg D. Compulsive hoarding. Am J Psychother 1987;41:409-416.

26. McElroy SL, Keck Jr. PE, Phillips KA. Kleptomania, compulsive buying, and binge-eating disorder. J Clin Psychiatry 1995;56:14-26.

27. Frost RO, Gross RC. The hoarding of possessions. Behav Res Ther 1993;31:367-381.

28. Frost RO, Hartl TL, Christian R, Williams N. The value of possessions in compulsive hoarding: patterns of use and attachment. Behav Res Ther 1995;33:897-902.

29. Damecour CL, Charron M. Hoarding: a symptom, not a syndrome. J Clin Psychiatry 1998;59:267-272.

30. Frost RO, Shows DL. The nature and measurement of compulsive indecisiveness. Behav Res Ther 1993;31:683-692.

31. Coles ME, Frost RO, Heimberg RG, Steketee G. Hoarding behaviors in a large college sample. Behav Res Ther 2003;41:179-194.

32. Frost RO, Krause MS, Steketee G. Hoarding and obsessivecompulsive symptoms. Behav Modif 1996;20:116-132.

33. Nisbett RE, Wilson TD. Telling more than we can know: verbal reports on mental processes. Psychol Rev 1977;84: 231-259.

34. Mann L. Decisional procrastination scale. Flinders University of South Australia; 1982.

35. Mann L, Burnett P, Radford M, Ford S. The Melbourne decision making questionnaire: an instrument for measuring patterns for coping with decisional conflict. J Behav Decis Making 1997;10: $1-19$.
36. Frost RO, Steketee G, Grisham J. Measurement of compulsive hoarding: Saving Inventory-Revised. Behav Res Ther 2004;42:1163-1182.

37. Foa EB, Huppert JD, Leiberg S, Langner R, Kichic R, Hajcak G, Salkovskis P. The obsessive-compulsive inventory: development and validation of a short version. Psychol Assess 2002;14: 485-496.

38. Beck AT, Steer RA, Ball R, Ranieri W. Comparison of Beck Depression Inventories -IA and -II in psychiatric outpatients. J Pers Assess 1996;67:588-597.

39. Beck AT, Steer RA. BDI, Back depression Inventory Manual. San Antonio, TX: Psychological Corp; 1987.

40. Spielberger CD, Gorusch RL, Lushene RE, Vagg PR, Jacobs GA. State and trait anxiety inventory for adults. Redwood City, CA: Mind Garden; 1977.

41. Steketee G, Frost R, Kyrios M. Cognitive aspects of compulsive hoarding. Cogn Ther Res 2003;27:463-479.

42. Effert BR, Ferrari JR. Decisional procrastination: examining personality correlates. J Soc Behav Pers 1989;4:151-156.

43. Ward JHJ. Hierarchical grouping to optimize an objective function. J Am Stat Assoc 1963;58:236-244.

44. Tolin DF, Frost RO, Steketee G. Buried in treasures: help for compulsive acquiring, saving, and hoarding. New York: Oxford University Press; 2007.

45. Vander Wall SB. Food hoarding in animals. Chicago: University of Chicago Press; 1990.

46. Wincze JP, Steketee G, Frost RO. Categorization in compulsive hoarding. Behav Res Ther 2007;45:63-72.

47. Kahneman D, Knetsch JL, Thaler RH. Experimental tests of the endowment effect and the Coase theorem. J Polit Econ 1990;98:1325-1348.

48. Thaler R. Towards a positive theory of consumer choice. J Econ Behav Organ 1980;1:39-60.

49. Frost RO, Steketee G, Williams L. Hoarding: a community health problem. Health Soc Care Community 2000;8:229-234.

50. Patronek GJ. Hoarding of animals: an under-recognized public health problem in a difficult-to-study population. Public Health Rep 1999;114:81-87.

51. United Nations Development Programme U. Consumption for human development. New York: Oxford University Press; 1998. 